\title{
Review
}

\section{Pathways in small cell lung cancer and its therapeutic perspectives}

\author{
Pingali M. Shivapriya ${ }^{1}$, Anirudh Singh ${ }^{1}$, Priyanshu Pandey ${ }^{2}$, Nandini Chhabra ${ }^{3}$, \\ Amaresh Kumar Sahoo ${ }^{1}$, Biswaranjan Paital ${ }^{4, *}$, Pritish Kumar Varadwaj ${ }^{1, *}$, Sintu Kumar Samanta ${ }^{1, *}$ \\ ${ }^{1}$ Department of Applied Sciences, Indian Institute of Information Technology Allahabad, 211012 Allahabad, India, \\ ${ }^{2}$ Department of Biotechnology, National Institute of Technology Durgapur, 713209 Durgapur, West Bengal, India, \\ ${ }^{3}$ Department of Studies in Biotechnology, University of Mysore, 570005 Mysore, Karnataka, India, ${ }^{4}$ Redox Regulation \\ Laboratory, Department of Zoology, Odisha University of Agriculture and Technology, College of Basic Science and \\ Humanities, 751003 Bhubaneswar, Odisha, India
}

\section{TABLE OF CONTENTS}

1. Abstract

2. Introduction

3. Challenges in SCLC treatment

4. Developmental signalling pathways in SCLC initiation and progression

4.1 Hedgehog signalling pathway

4.2 NOTCH signalling pathway

4.3 Fibroblast Growth Factors signalling pathway

4.4 Other developmental signalling pathways in SCLC

5. Role of immunotherapy on signalling pathways

6. Conclusion and future prospects

7. Author contributions

8. Ethics approval and consent to participate

9. Acknowledgment

10. Funding

11. Conflict of interest

12. References

\section{Abstract}

Small cell lung cancer (SCLC) is known for its rapid growth with high metastatic spread. Its treatment remains a major challenge for oncologists due to the high mutation rate and other clinical disadvantages. The survival rate of these patients is very poor but there is no significant progress over the last few decades in the treatment protocols. Hence there is an urgency to design new clinical trials with novel drug combinations that can specifically and effectively target key pathways for proper treatment of SCLC. There have been several indications that developmental signalling pathways are involved in tumor growth, progression, metastasis and invasion of SCLC. Thus, it is anticipated that deciphering the signalling cascades of these embryonic signalling pathways may reveal novel therapeutic breakthroughs in SCLC. In this context, we have tried to assemble all the relevant information to give an updated overview of the various signalling pathways involved in the development of SCLC.

\section{Introduction}

Lung Cancer is one of the leading causes of death across the world [1]. Based on histology, lung cancer can be classified into two types: small cell lung cancer (SCLC) and non-small cell lung cancer (NSCLC). SCLC accounts for around $14 \%$ of all lung cancers [2] and each year, around 30,000 people are diagnosed with SCLC [3]. Tobacco exposure remains the primary risk factor and this risk increases with the intensity and duration of tobacco usage [4]. This cancer originates from the pulmonary neuroendocrine cells (PNECs) or Kulchitsky cells (K cells) and is associated with mutation-induced loss of function of the tumor suppressor genes like Rb and Trp53 [5]. SCLC shows early metastasis to various organs like adrenal glands, brain, bone, lymph nodes, and liver. Both the rapid growth and high metastatic nature results in a high mortality rate in patients suffering from SCLC [6]. The survival chances barely increase after undergoing treatment. After initial treatment, less than $5 \%$ of the patients could survive for more than 5 years and 
this period is even brief for treatment-naive patients with an average overall survival period of only 2-4 months [7]. Also, the standard treatment strategy for SCLC has not been changed in the last few decades [8].

Based on the extent to which cancer had spread from its primary site, SCLC can be staged as an Extensive stage disease (ED) and Limited stage disease (LD). Generally, we call it LD when the tumor is confined to one lung and if it spreads beyond that, it is said to be ED. Generally, both the LD and ED patients give a satisfactory response to initial chemotherapy and radiotherapy but most of them show a relapse in 6-12 months [9]. The LD-SCLC patients are normally treated with 4-6 cycles of chemotherapy with carboplatin or cisplatin and etoposide along with thoracic radiotherapy [10]. For the ED-SCLC patients, combined chemotherapy of carboplatin plus etoposide is administered that yields a satisfactory response in $60-70 \%$ of patients. Nearly $10 \%$ of these patients showed a complete radiographic response to initial chemotherapy and are subsequently considered for PCI and/or thoracic radiotherapy [10]. Importantly, prophylactic cranial irradiation (PCI) and chest irradiation (CRT) were successful in increasing the survival rate $[6,11]$. Prophylactic Cranial Irradiation (PCI) is generally recommended for those who demonstrate a satisfactory response to the initial therapy. It was noted that $90 \%$ of the patients respond to such treatment while $25 \%$ of them gain long-term survival [10]. Since the development of active chemotherapy regimens 4 decades ago, the overall survival of SCLC patients had slightly improved. The 2-year survival rate of LD-SCLC patients has been improved from 15 to $22 \%$ with a slight increase (3.4 to 5.6\%) for ED-SCLC patients during the period of 1973-2002 [10]. Over the last 30 years, a number of treatment protocols have failed to treat ED-SCLC. After the approval for Topotecan as second-line treatment, it took more than 20 years to get FDA approval for Nivolumab in 2018 [12].

Various developmental signalling pathways like Notch, hedgehog play an important role in cellular functions including cell motility, lineage, apoptosis, etc. It has been observed that these pathways also drive the initiation and maintenance of SCLC cells [13]. These pathways are very crucial in regulating self-healing in case of injury [14]. Thus, giving an indication that misregulation of these pathways may contribute to tumor growth. These characteristics have paved a path to explore these pathways as potential drug targets for cancer treatment. In this article, we have highlighted several challenges that are faced by the research fraternity to propose a new treatment for SCLC. Also, it provides a first comprehensive review on the roles of various developmental pathways for the initiation and growth of SCLC along with the attempts made by various research groups to reduce the progression of this cancer by targeting these pathways.

\section{Challenges in SCLC treatment}

For the last 15 years, there has been no improvement in survival rates and no new treatment has been approved. Here, we have highlighted some major issues that make the treatment difficult and challenging for SCLC.

No early diagnostic method: Due to the rapid growth and early metastatic spread, most of the SCLC patients are diagnosed at a later stage when the disease gets widespread. As per the report of the National Lung Screening Trial conducted in 2002, early-stage SCLC could not be detected by CT scan. It is very unfortunate that no effective diagnostic method is available to date for the early detection of SCLC [15].

Non-availability of sufficient tumor tissue for diagnosis and study of SCLC: Only a very small amount of tumor tissues like fine-needle aspirates, core biopsies, and bronchoscopic biopsies are available to perform the translational research to develop the diagnosis and treatment process of SCLC. Also, the small cell size makes the handling of these cells difficult as they can be easily distorted using forceps or aspiration needles used during sample collection [16]. Though the majority of the SCLC patients are treated by chemotherapy and radiotherapy, a small number of patients undergo surgery to remove the tumor. Since it requires robust materials for analyzing molecular profiling like genome or exome sequencing, the non-availability of tissues limits such study for SCLC. Therefore, it was not possible to include SCLC in The Cancer Genome Atlas (TCGA) [15].

The paucity of animal models: The nonavailability of animal models also limits the study on SCLC. There are only a few genetically engineered mouse models available. However, none of these models can fully mimic the genetic complexity of SCLC in humans. It is basically due to the lower mutational burden for the non-exposure of tobacco to the animal model. These models are very costly and demand a great deal of time for the development of cancer. It requires more than 9 months for developing models with inactivated Trp53 and Rb1 [17]. In addition, it was noticed that during the transfer of cancer cells from one xenograft model to another, certain mutations that were present in the parent cell line were found missing in the transferred model [15].

Complex molecular pathogenesis of SCLC: Due to high exposure to tobacco carcinogens, the molecular pathology of SCLC becomes highly complex with lots of genetic alterations like insertions, deletions, mutations, gross inter-and intra- chromosomal rearrangements, and large-scale copy number alterations. Most of the mutations obtained in SCLC are passenger mutations indicating that these don't significantly contribute to the growth and progression of the disease. Since only a few mutations are identified in all the cases, it gives very few therapeutic targets for the treatment. It was noticed that the mutations of TP53 and RB1were found in 75-90\% and 60-90\%, cases respec- 
tively. The underlying mechanistic details that contribute to the elevated metastatic behaviour of SCLC are still unknown [15].

Resistance to chemotherapy: The majority of LDSCLC and ED-SCLC patients experience a relapse in just 6-12 months after completion of initial treatment. Both the molecular mechanisms involved in the development of resistance and the chemoresistant properties of the cell population are yet to be discovered [15].

Lack of SCLC-specific research projects: The research work with SCLC has been dramatically decreased for the lack of tumor tissue and animal models. It was clearly reflected on the National Cancer Institute (NCI) research portfolio that considers only 17 projects (approx. 2\%) for SCLC out of 745 projects on lung cancer research [15].

\section{Developmental signalling pathways in SCLC initiation and progression}

Developmental signalling pathways are crucial for proper embryonic development, are highly conserved and expressed in most multicellular organisms [18]. The deregulation of these pathways may lead to tumor initiation and progression among adults $[18,19]$. In the case of various tumors, the misregulation of these pathways may also lead to metastasis, drug resistance, and angiogenesis [19]. Accordingly, these pathways are gaining importance as potential drug targets especially in the case of advanced and metastatic tumors. SCLC is a rapidly growing tumor with a very quick stage transition time. Furthermore, in most cases, this type of cancer is identified in the patients when it has already reached its extensive stage (ED). In addition, the tumor cells become resistant to chemotherapy even after the initial treatment [20]. Vowing to all these factors, here we have tried to provide an understanding of the roles played by the developmental signalling pathways for initiation and progression of SCLC. We have also briefly mentioned the efforts that have been made in this respect to counter this deadly tumor.

\subsection{Hedgehog signalling pathway}

Hedgehog (Hh) signalling pathway has a central role in controlling the cell fate, tissue polarity, and organogenesis during development [21]. The Hh pathway is highly regulated in adult tissues under normal conditions. It can also play a vital role in early lung development by epithelial-mesenchymal interactions [21, 22]. However, in the case of cancer, it is observed that there is a misregulation in this pathway. The canonical pathway can be activated by Hh glycoproteins like Sonic (Shh), Indian (Ihh), and Desert (Dhh) that act as ligands for the Hh pathway. Among these, Shh is the most potent ligand [23]. When the Hh ligands are absent, the Patched1 (PTCH1), a 12pass transmembrane receptor inhibits the progression of the pathway. Further, the binding of Shh to PTCH1 re- lieves the repression of Smoothened (SMO) protein that helps in its translocation into the primary cilium. This helps in the activation of glioma-associated transcription factors (GLI1, GLI2, GLI3) via initiation of the signalling cascade. When SMO is inactive, GLI is bound to Suppressor of fused (SUFU) due to which GLI remains in an inactive state. As a result, this complex gets accumulated in the cytoplasm which ultimately results in its degradation [24]. In the active state, the GLI dissociates from the SUFU and is transported to the nucleus where it regulates the transcription of the associated genes (Fig. 1A) [25].

\subsubsection{Role of Hedgehog pathway in SCLC}

During the development of the lung, Hh signalling pathway is crucial for the regulation of branching morphogenesis in a paracrine manner. However, in normal adult lungs, this signalling pathway gets reactivated during severe tissue damage [22] and becomes necessary for the stem cell compartments [26]. In the case of SCLC, it is found that some of the cells express Shh while the adjacent cells express PTCH1. This indicates that similar to lung development and repair, SCLC cells also have an active Hh signalling in a paracrine manner [27]. It was supported by the fact that there was a significant reduction in the SCLC growth upon treatment with 5E1 Shh-N monoclonal antibody [22] indicating the importance of this pathway in tumor growth. However, there is no evidence of the change in copy number of the hedgehog genes till date [28]. In a study by Park and his collaborators, it was found that Hh signalling plays an important role in SCLC right from its initiation to its maintenance indicating that both initial and advanced stages of SCLC tumors are dependent on an active Hh signalling [13]. In this study, it was observed that upon deletion of SMO in Trp53 and Rb1 negative lung mutant cells, the initiation of SCLC was suppressed. Also, they could successfully inhibit the tumor growth post-chemotherapy, in both human and mouse models by inhibiting the Hh pathway.

\subsubsection{Therapeutics targeting Hedgehog pathway in SCLC}

As already discussed, the Hh pathway plays a crucial role in SCLC right from its initiation to its development, it has been used as a potential target. The majority of the studies are directed against the SMO protein that plays a vital role in activating the Hh pathway. Cyclopamine is one such compound that selectively binds to SMO to inhibit its function by causing its conformational change which is very similar to that caused by PTCH1 activity [29] (Table 1). Vismodegib, a novel small molecule inhibitory drug that is directed against SMO is widely used to inhibit the Hh pathway after noticing its promising results in the case of advanced Basal cell carcinoma [30] (Table 1, Ref. [29, 3144]). However, there was no significant improvement observed in the ED-SCLC patients when Vismodegib was administered along with standard chemotherapy in ED-SCLC [31]. Apart from this, the SMO inhibitors namely Eris- 
B

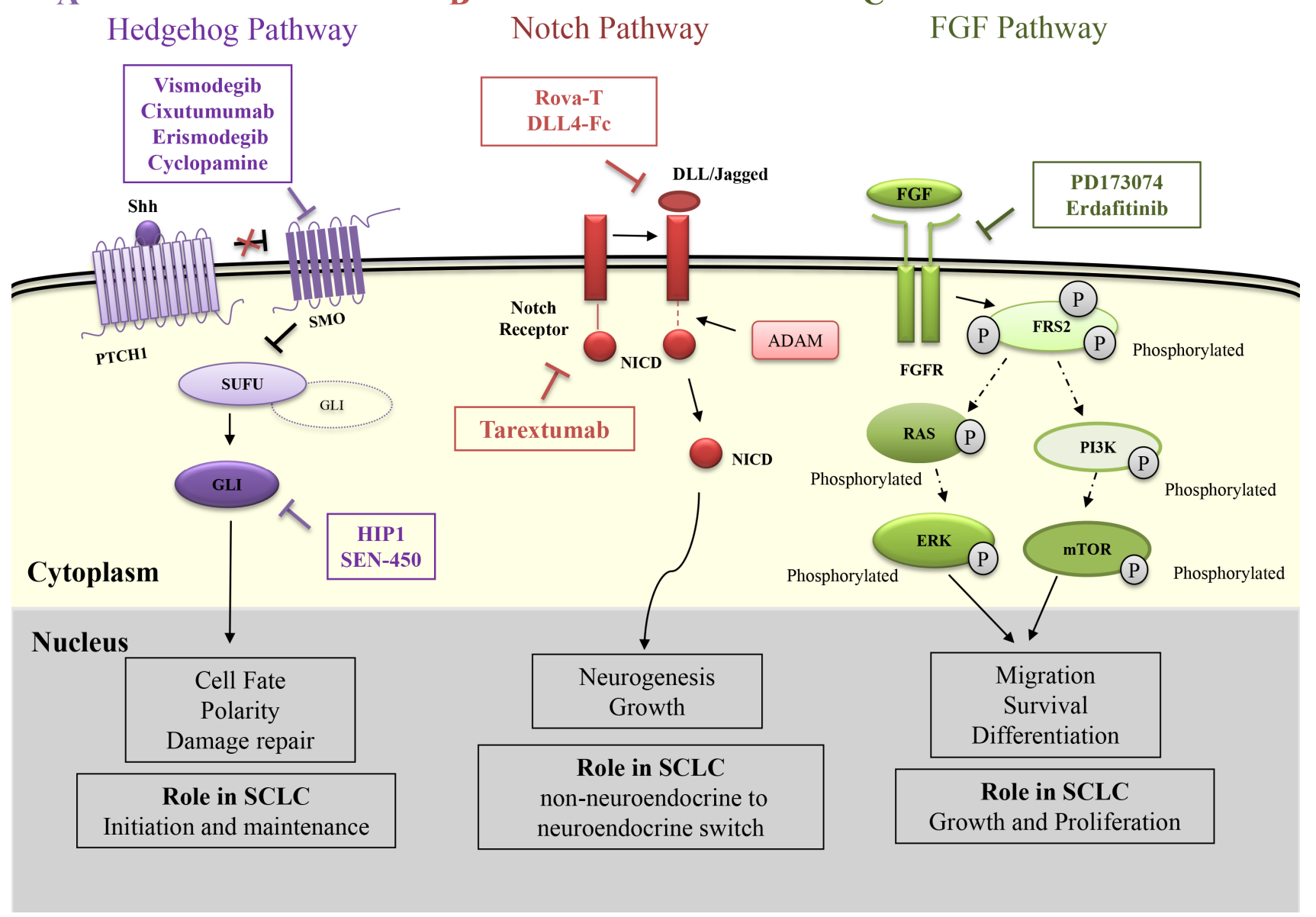

Fig. 1. Role of Hedgehog, Notch and FGF signalling pathways in SCLC and their therapeutic interventions. (A) Hedgehog pathway - The pathway is initiated when the ligand shh binds to the patched1 receptor (PTCH1). This relieves the inhibition on the Smoothened (SMO) protein that is responsible for inhibiting Suppressor of Fused (SUFU). SUFU prevents the entry of Glioma associated Transcription Factor (GLI) into the nucleus when activated. Thus, SUFU inhibition releases the bound GLI into the cytoplasm after which it is transported to the nucleus and acts as a transcription factor. The therapeutics Vismodegib, Erismodegib, Cyclopamine and SEN450 inhibits the SMO function while HPI1 inhibit the GLI. (B) Notch Pathway - The binding of Delta like ligand (DLL) or Jagged ligand activates ADAM and several other proteins that cleaves the Notch intracellular domain (NICD) from the notch receptor. The NICD then moves to the nucleus and helps in the expression of Notch related genes. The therapeutics Rova-T and DLL4-Fc inhibits the DLL ligand while Tarextumab inhibits the notch receptor. (C) FGF pathway - The Fibroblast growth factor receptor (FGFR) is activated when FGF is bound to it. FGFR then phosphorylates Fibroblast Growth Factor Receptor Substrate 2 (FRS2) at multiple sites. This initiates several signalling cascades where Ras and Phosphoinositide 3-kinases (PI3K) being the prominent ones. Their end effectors ERK and mTOR help in transcription of target genes. The therapeutics against this pathway PD173074 and Erdafitinib target the FGFRs.

modegib and Vismodegib did not show any significant results on 23 different SCLC cell lines when given along with chemotherapy. Similar findings were observed even with the treatments of HPI1 and SEN450, specific inhibitors for GLI and SMO respectively (Table 1 ). The compounds erismodegib, HPI1, and SEN450 showed a better result in the case of MYC amplified cell lines [32] indicating a critical role of MYC in this mode of treatment (Fig. 1A).

\subsection{NOTCH signalling pathway}

The NOTCH signalling pathway was initially observed and identified by Otto L. Mohr in Drosophila melanogaster [45]. NOTCH signalling is actively involved in embryonic development with overgrowth of the nervous system. The canonical NOTCH signalling pathway is initiated when the ligand belonging to the delta-like (DLL) or Jagged family proteins binds to the NOTCH receptor [46]. This results in an enzymatic cleavage of the receptor by Adisintegrin and metalloprotease (ADAM) followed by a final breakage by the presenilin-g-secretase complex. This causes the dissociation and translocation of the intracellular part of the NOTCH receptor - NOTCH intracellular domain (NICD) to the nucleus [47]. In the nucleus, the NICD binds with other transcription factors (TFs) and promotes gene expression of the Hey and Hes family of genes along with other genes related to VEGF, NFkB, BcL etc. [48]. The Hes and Hey family of genes regulate the differentiation of the lung epithelial cells by inhibiting the transcrip- 
Table 1. Therapeutics that have been tested against SCLC that target developmental signalling pathways.

\begin{tabular}{|c|c|c|c|c|c|}
\hline S. No & Name & Target pathway & Mechanism of Action & Current Status in SCLC & References \\
\hline 1 & Cyclopamine & Hedgehog & $\begin{array}{l}\text { It inhibits SMO by causing a confirmational } \\
\text { change that is similar to the PTCH1 activity } \\
\text { when the Hh ligand is present. }\end{array}$ & Not enrolled in clinical trials & [29] \\
\hline 2 & Vismodegib & Hedgehog & $\begin{array}{l}\text { Acts as SMO antagonist and inhibits the } \\
\text { downstream signalling of the Hh pathway. }\end{array}$ & Phase 2 completed & {$[31,39]$} \\
\hline 3 & Erismodegib & Hedgehog & $\begin{array}{l}\text { SMO antagonist that can induce apoptosis } \\
\text { in cancer cells. }\end{array}$ & Phase 1 completed & {$[40,41]$} \\
\hline 5 & HPI1 & Hedgehog & $\begin{array}{l}\text { Induces structural changes on GLI that } \\
\text { inhibit the gene expression in SUFU } \\
\text { deactivated or GLI overexpressing cells. }\end{array}$ & Not enrolled in clinical trials & {$[32,42]$} \\
\hline 6 & SEN450 & Hedgehog & SMO antagonist. & Not enrolled in clinical trials & {$[32,43]$} \\
\hline 7 & DLL4-Fc & NOTCH & $\begin{array}{l}\text { Reduces Notch1 signalling in DLL4 } \\
\text { expressing cells. This halts the early } \\
\text { metastasis of cancer cells. }\end{array}$ & Not enrolled in clinical trials & [33] \\
\hline 8 & $\begin{array}{l}\text { Tarextumab } \\
\text { (OMP-59R5) }\end{array}$ & NOTCH & $\begin{array}{l}\text { Targets and inhibits the Notch2/3 signalling. } \\
\text { It is a human IgG antibody specific for } \\
\text { Notch receptors. }\end{array}$ & Terminated in Phase 1b & [34] \\
\hline 9 & $\begin{array}{l}\text { Rova-T } \\
\text { (Rovalpituzumab } \\
\text { Tesirine) }\end{array}$ & NOTCH & $\begin{array}{l}\text { It is an antibody-conjugated drug that targets } \\
\text { DLL3. Once bound, the linker is cleaved } \\
\text { releasing the drug into the cytoplasm. The } \\
\text { drug enters the nucleus and binds to the } \\
\text { DNA leading to cell cycle arrest. }\end{array}$ & Phase 3 completed & {$[35,44]$} \\
\hline 10 & Erdafitinib & FGF & $\begin{array}{l}\text { Binds and inhibits the function of FGFRs. } \\
\text { Mainly inhibits the phosphorylation which } \\
\text { ultimately inhibits the downstream } \\
\text { signaling. }\end{array}$ & Not enrolled in clinical trials & [37] \\
\hline 11 & PD173074 & FGF & Binds and inhibits the function of FGFRs. & Not enrolled in clinical trials & [36] \\
\hline 12 & XAV939 & WNT & $\begin{array}{l}\text { Downregulates } \beta \text {-catenin which reduces the } \\
\text { growth of cyclin D1. }\end{array}$ & Not enrolled in clinical trails & [38] \\
\hline
\end{tabular}

tion of basic helix-loop-helix proteins such as achaete-scute homologue 1 (ASCL1) [49, 50]. This transcription factor is responsible for the development of the precursor cells for SCLC (Fig. 1B).

In mammals, there are four variants of NOTCH receptors: NOTCH-1, NOTCH-2, NOTCH-3, and NOTCH4. The delta-like ligand proteins are mainly of 3 variants (DLL1, DLL3, and DLL4) [51] whereas the Jagged ligand proteins are of two variants: Jagged-1 (JAG-1) and Jagged-2 (JAG-2) [52]. These ligands can bind to the receptor within the same cell (cis-interaction) or with that of the different cell (trans-interaction) which causes inhibition or activation of the signalling pathways, respectively [53]. Among these various ligands, DLL3 is the only ligand that makes a cis interaction with the receptor [54]. Also, unlike the other DLL receptors, DLL3 does not activate the notch pathway. It usually inhibits both the cis and trans activating Notch signalling pathway by binding to the notch and DLL1 and restricting their accumulation on the cell membrane [55]. DLL3 is highly expressed in the fetal brain and is also a direct downstream target of the ASCL1 which is a key player for neurogenesis [55]. This suggests that DLL3 can be crucial for neuroendocrine tumorigenesis.

\subsubsection{Role of NOTCH signalling in SCLC progression}

NOTCH signalling can be both tumours suppressive and promoting based on the type of cell and the context. In SCLC, the activation of NOTCH signalling has a tumorsuppressive role [56]. It is found that the majority of neuroendocrine features in SCLC are due to NOTCH inactivation [57]. The NOTCH signalling may interfere with important functions of the cell-like apoptosis, mesenchymal differentiation, and neuroendocrine differentiation which help in tumor progression and metastasis in SCLC. The role of NOTCH signalling in apoptosis was first established by Sriuranpong and his group [58]. They have observed a significant growth inhibition when they transfected SCLC with active NOTCH1/2. This was because of the G1 cell cycle arrest that was trigged due to the selective activation of p21. However, this is not a common phenomenon in all cell types and is reported only in the case of SCLC. Another study by Hassan et al. [59], also achieved successful inhibition of SCLC tumor growth by Bcl-2 inhibition. Here, NICD was transfected to H69 and H1688 SCLC cells that activated apoptosis by inhibiting Bcl-2 [60]. On the contrary, NOTCH1 transfection in H69AR and SBC-3 cells enhances the expression of Bcl-2 but inhibits apoptosis [59]. The NOTCH pathway is also crucial for epithe- 
lial to mesenchymal transition (EMT) of the SCLC cells. This transition is important for the ability of SCLC cells to invade and metastasize to other locations in the body. It was also found that NOTCH knockdown promoted the expression of several EMT-related proteins in SCLC cells $[59,61]$. NOTCH1 reactivation promoted the cell motility and invasion by inhibiting gamma laminin 2 chain alpha [59, 61]. Similar results were also observed for the case of NOTCH3 [61]. Also, in a study conducted by Meder and collaborators, it was found that just mutation can inactivate NOTCH which is sufficient for non-neuroendocrine to neuroendocrine switch in tumor cells or precursors [62].

\subsubsection{Therapeutics targeting NOTCH pathway in SCLC}

In spite of its tumor-suppressive role in SCLC, NOTCH activation may lead to other complications in regard to the development of chemoresistance of the SCLC tumor. This is due to the fact that the NOTCH pathway induces intratumoral heterogeneity in SCLC. NOTCH activation seems to be central in triggering the switch from neuroendocrine to non- neuroendocrine fate [63] leading to increased chemoresistance [64]. The study led by Lim et al. [63] demonstrates that non-neuroendocrine cells having active NOTCH signalling were slow-grower and showed tumor-suppressive role; however, these cells showed more resistance to cisplatin plus etoposide compared to the neuroendocrine. The above findings emphasize the intricacy of targeting NOTCH signalling to design the most appropriate treatment against SCLC.

A pre-clinical study conducted using DLL4-Fc, a DLL4 inhibitor, gave positive results in reducing the liver metastasis of SCLC in murine models [33]. This gave initial evidence of a positive therapeutic consequence by modulating the NOTCH pathway in SCLC (Table 1). By keeping in mind, the heterogeneity of SCLC, compound Tarextumab (OMP-59R5), a complete human IgG2 antibody was used in combination with chemotherapy in order to prevent the switch of chemosensitive neuroendocrine cells to chemoresistant non-neuroendocrine cells (Table 1). This drug was specific for targeting NOTCH2 and NOTCH3 receptors, and its efficacy was analyzed in SCLC allografts followed by patient-derived xenografts (PDX) and then in SCLC patients [34] (Fig. 1B). As expected, Tarextumab combined with carboplatin plus irinotecan gave better results in comparison to Tarextumab or other chemotherapy used individually. However, this compound did not reach its primary goal of progression-free survival [34]. It was also observed that DLL3, the inhibitory ligand of the notch pathway was overexpressed in the case of SCLC [35, 65]. Thus, an antibody-drug conjugate called Rova-T (Rovalpituzumab Tesirine) was designed to target DLL3 (Fig. 1B). The results of Rova-T were encouraging in phase- 1 trials which gave a very good response with minimal toxicity [65]. However, the same could not be observed in the later trials [66] (Table 1).

\subsection{Fibroblast Growth Factors signalling pathway}

The Fibroblast Growth Factors (FGF) have an important role in the proliferation, survival, differentiation, and migration of cells in both embryonic and adult stages [67]. Their signalling is mediated by specifically binding to the fibroblast growth factor receptor (FGFR) which belongs to the family of tyrosine kinase receptors (RTKs). There exist 4 FGFR receptors: FGFR1, FGFR2, FGFR3, and FGFR4. They are single-pass transmembrane proteins consisting of the intracellular domain, extracellular domain and transmembrane region [68]. Upon activation, FGFR phosphorylates Fibroblast Growth Factor Receptor Substrate 2 (FRS2) at different sites that further activates Ras and downstream Mitogen-activated protein kinases (MAPKs) and RAF with the help of growth factor receptor-bound protein 2 (GRB2) and adaptor proteins [69]. The intracellular receptors like FRS2 and phospholipase C (PLC) further mediate the downstream signalling process that eventually up-regulates RAS-independent phosphoinositide 3kinase-AKT (PI3K/AKT) and Ras-dependent MAPK pathway which is highly crucial for progression of the cell cycle [67]. The activation of phospholipase C leads to the hydrolysis of phosphatidylinositol-4, 5-bisphosphate (PIP2) to phosphatidylinositol-3, 4, 5-triphosphate (PIP3) and diacylglycerol [70]. This activates protein kinase C (PKC) which phosphorylates RAF in order to activate the MAPK pathway. Many other pathways including p38 MAPK and Jun $N$-terminal kinase (JNK) pathways are triggered by FGFRs [71]. The activation of the above pathways regulates motility and proliferation, cell survival, invasiveness, epithelial-to-mesenchymal transformation (EMT), and angiogenesis (Fig. 1C). Generally, FGFs and FGFRs are expressed in several tissues of mesenchymal and epithelial origin. They play a key role in angiogenesis along with the formation and growth of different organs in embryonic life [72]. Signalling cascades mediated through FGF/FGFR play a pivotal role in tissue homeostasis, inflammatory processes, and vessel maturation during tissue and wound healing [67].

\subsubsection{Role of Fibroblast Growth Factors in SCLC}

Integrated genome analysis showed FGFR1 focal amplification in $6 \%$ of SCLC cases [73]. Studies performed on the SCLC cell lines and tumors have reported the occurrence of high copy number gains (CNGs) in the FGFR1 cytogenetic bands $[28,73]$ which indicate the possibility of FGFR1 as a therapeutic target for SCLC [74]. Inhibition of FGFR weakens the growth of SCLC, decreases intratumor proliferation, and increases apoptosis indicating that FGFR can influence SCLC growth [36]. Pardo et al. [36] demonstrated the dose-dependent decrease of proliferation in human SCLC xenograft models (H-510 and H-69) with the oral administration of FGFR inhibitor, PD173074 for 28 days. Interestingly, the longer median survival was obtained in H-150 xenograft as compared to control animals by the combined treatment of cisplatin and PD173074. 
Both the SCLC cell lines and tumor samples demonstrate the focal amplifications and FGFR1 mutations in 33.3\% of cases indicating that FGFR1 could be one of the effective therapeutic targets [75]. In accordance, a recent study has demonstrated the positive FGFR1 gene copy numbers or FGFR1, FGF2, FGF9 protein in a subset of SCLC patients. We need to perform further studies in order to analyze the efficacy of FGFR specific inhibitors in the large population of SCLC patients.

\subsubsection{Therapeutics targeting Fibroblast Growth Factors pathway in SCLC}

There is strong evidence suggesting that the improper activation of the FgFr pathway has a tumorigenic role in the body. This pathway is responsible for some of the key characteristics of the tumor-like angiogenesis and drug resistance [76]. FgFr amplification is believed to be a rare event in SCLC; it has been observed that this amplification is associated with a shorter disease-free survival time after the first-line chemotherapy [76]. However, a strong conclusion on this aspect can't be made due to the lower sample size of the patients and/or shortage of the tissue. The preliminary studies indicate that small molecule inhibitors targeting FgF receptors can be used as drugs to inhibit the signalling cascade [77]. Erdafitinib being the first selective compound against FgFr was approved by FDA in 2019 [37] (Table 1). In the case of SCLC, PD173074 administered along with chemotherapy showed a longer survival in mouse xenografts [36] (Table 1). The FgFr signal inhibition has already shown a good anti-tumorigenic response in other cancers including non-small cell lung cancers [77]. However, there is a lot to be known before we can propose FgFr as a possible drug target for SCLC. The difficulties in the process of tissue samples collected for the aggressive nature of the disease is the underlying cause for the lack of accepted therapies [78] (Fig. 1C).

\subsection{Other developmental signalling pathways in SCLC}

As already mentioned, there is no clear understanding of how SCLC progresses in the human body. Thus, there is still a lot more that needs to be identified as to which developmental pathways are active during the development of this cancer. Most of the research in this area is concentrated on the above-mentioned pathways. However, there is some evidence for the activation of other developmental pathways as well. For example, RNA-sequencing data revealed that the WNT signalling pathway was mutated predominantly in the case of SCLC patients with recurrent disease [79]. However, this study was performed on a very small population of 12 patients who have previously undergone treatment with platinum chemotherapy. Thus, it can't be clearly said if these mutations are a part of disease progression or they are generated due to the treatment [80]. Prior to this study, the evidence for activation of WNT signalling in SCLC was indirectly given by the overexpression of the SOX-2 gene that in turn regulates the expression of certain oncogenes like n-MYC, WNT-1, WNT-2, and
NOTCH1 in human lung adenocarcinomas [81]. Further, there was a clear indication that downregulation of SOX-2 decreased tumorigenesis and induced apoptosis [81]. Thus, it can be inferred that SOX-2 is responsible for the activation of pathways like WNT and NOTCH which in turn induce tumorigenesis. A study by Pan and his group have demonstrated that XAV939, a small molecule inhibitor of the WNT signalling pathway could significantly inhibit the proliferation of the SCLC in vitro [38] (Fig. 2A).

The transforming growth factor- $\beta$ (TGF- $\beta$ ) signalling pathway is reported to be upregulated in various pulmonary diseases [82]. This pathway inhibits earlystage tumor progression but in later stages, it promotes tumor growth. In the case of SCLC, the inhibition of this pathway reduced the migration and invasion of the cancer cells [83]. This was confirmed by the downregulation of miR-886-3p miRNA which is in turn responsible for the post-transcriptional suppression of TGF- $\beta 1$ and PLK1 [84] (Fig. 2B).

The overexpression of WW domain-binding protein 5 (WBP5) further indicates the involvement of another developmental pathway like Hippo in SCLC [85]. Activation of this pathway resulted in multidrug resistance, enhanced tumor growth, and shorter survival in SCLC patients. Later on, it was revealed that this resistance was mediated by the Hippo pathway through the inhibition of phosphorylation of key proteins like MST2 (mammalian Ste20-like kinase 1) and YAP1 (Yes-associated protein -1) [86]. This supports the idea that the Hippo pathway plays a role in developing multidrug resistance in SCLC. Recent research also showed that the Hippo pathway plays a role in radiation resistance in SCLC patients. It was found that MST2 had no significant role but YAP1 along with CD133 had a profound role in developing radiation resistance in SCLC [87] (Fig. 2C).

These findings clearly show that developmental signalling pathways are involved in key aspects of tumorigenesis in SCLC progression and also contribute to its neuroendocrine behavior. However, there are lots of grey areas that need to be addressed properly in order to consider the components of these pathways as drug targets.

\section{Role of immunotherapy on signalling pathways}

In recent times, cancer treatment using immunotherapy is gaining importance [88]. The use of immunotherapy in SCLC is employed alongside etoposide or other chemotherapeutic agents that are used for SCLC treatment. The immunotherapy treatment through PD-L1 inhibition is the current method for SCLC treatment. Studies indicated the cross-talk of this signalling with developmental signalling pathways like hedgehog and WNT in cancers like breast cancer and gastric cancer. In triple-negative breast, it was observed that WNT inhibitors play a role in the downregulation of PD-L1, while the Hedgehog pathway favours 


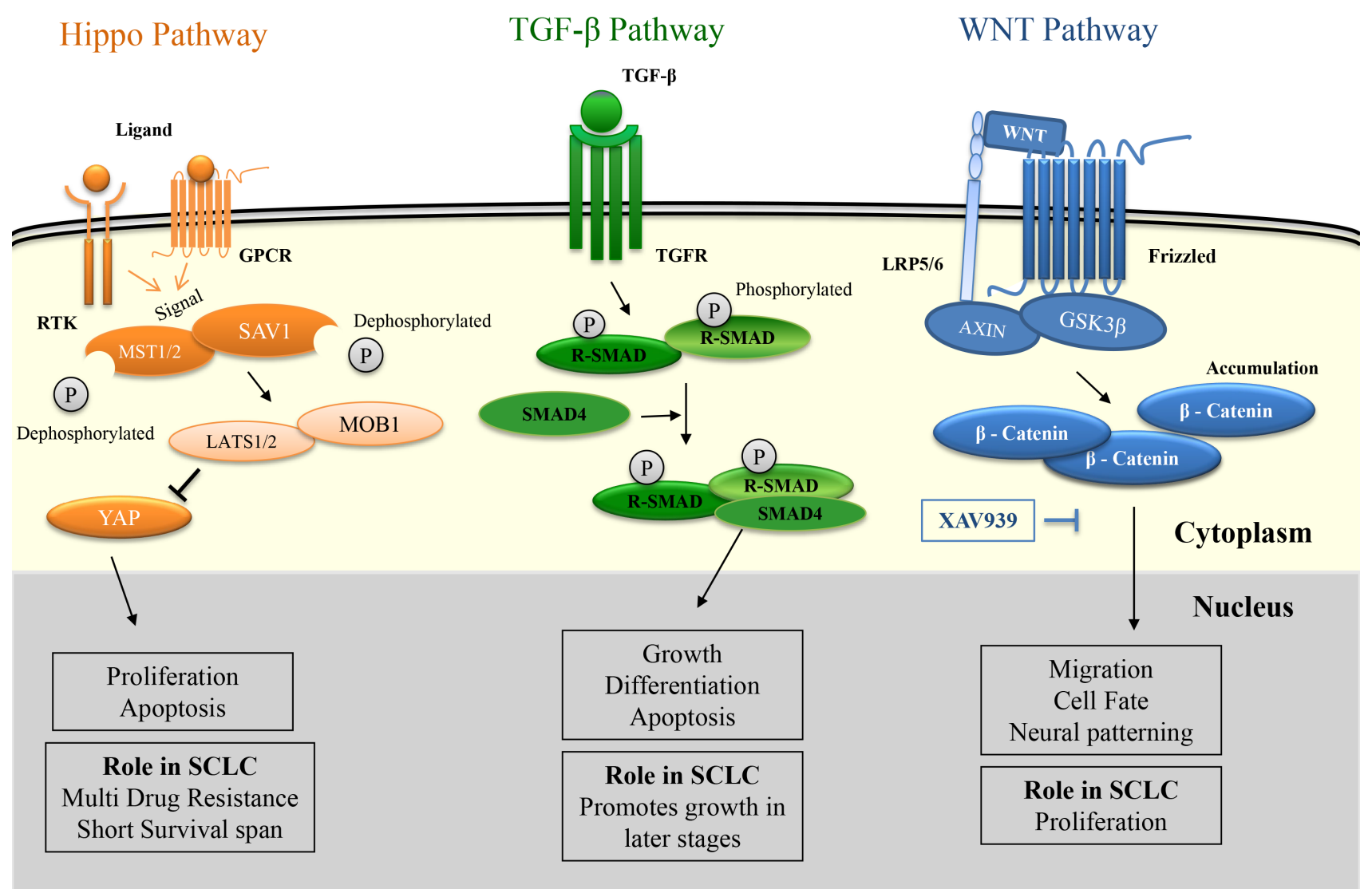

Fig. 2. Role of Hippo, TGF- $\beta$ and WNT pathways in SCLC and their therapeutic interventions. (A) WNT pathway - The WNT ligand when bound to the frizzled receptor and LRP5/6 translocates Axin and GSK- $\beta$ towards the plasma membrane. This results in accumulation of $\beta$-Catenin in the cytoplasm and then its transport to the nucleus where it regulates the gene expression. The transport of $\beta$-Catenin to the nucleus is prevented by XAV939, a small molecule inhibitor of WNT pathway. (B) TGF- $\beta$ Pathway - The ligand TGF- $\beta$ binds to the receptor TGFR that activates it. The activated receptor phosphorylates receptor mediated SMADs (R-SMAD) that binds with SMAD4 to function as a transcription factor. (C) Hippo pathway - The ligand binding at the Receptor Tyrosine Kinases (RTK) and G protein-coupled receptors (GPCR) releases a signal that inhibits phosphorylation of proteins like MST1/2, SAV1, LATS1/2, MOB1. This releases the LATS1/2 and MOB1 complex inhibiting yes-associated protein 1 (YAP) that is a transcription factor can regulate important cellular functions like apoptosis.

the expression of PD-L1 in gastric cancer [89, 90]. However, the exact mechanism by which these pathways are interrelated is not yet known. In addition, immunotherapymediated regulation of signalling pathways in the case of SCLC is also need to be deciphered.

\section{Conclusion and future prospects}

SCLC is a lethal malignancy with a rapid doubling time. There is no complete understanding of how this cancer initiates and progresses in the body. The patients respond well to initial therapy, but majority of them show relapse within a few months' time. Etoposide and topotecan along with platinum-based chemotherapy are being used as the first-line and second-line drugs respectively since 1970's. There has been no significant improvement in the treatment strategies over few decades. However, recent improvement in the radiation technology has modestly increased the survival rate for both limited and extensive stage SCLC. Although molecular anomalies associated with SCLC are known to the oncologists, efforts to target these aberrations over a decade have been consistently unsuccessful resulting in dismal prognosis for SCLC. As a result, SCLC has been enlisted along with other difficultto-treat cancers in the Recalcitrant Cancer Research Act of 2012 instructing National Cancer Institute (NCI) to allocate funds to deal with it. There is always a new hope that the improved understanding of the underlying molecular mechanisms may result in development of new therapeutics for SCLC. Thus, there is a need to identify more effective targets and also gain knowledge about the progression of this cancer. In this respect, research on the developmental signalling pathways has gained importance. There have been some interesting outcomes that demonstrate the importance of these pathways in initiation, growth, metastasis, inva- 
sion and progression of SCLC. The NOTCH and Hedgehog pathways were the most explored developmental pathways in SCLC. In other types of cancers, it has been observed that developmental pathways like FGF, Hippo, TGF- $\alpha$ and WNT had a profound role in tumorigenesis. But these pathways have not been studied extensively in SCLC. Preliminary studies gave some positive results in controlling the tumor growth. However, these compounds have not yet been tested in humans or if tested, the sample size of patients was very small to draw any conclusions from the study. Hence, we believe that an in-depth study in these developmental signalling pathways can throw more light into the progression and initiation of SCLC. Also, the crosstalk among these pathways can help us gain a better understanding about the neuroendocrinal switch and metastatic features of SCLC. Therefore, it is very much necessary to decipher the signalling cascades of the developmental pathways in more detail to find potent drug targets for successful treatment of SCLC. In view of the above, the current article provides the first comprehensive review about the available information on the role of developmental pathways in SCLC which can ultimately help in better prognosis and treatment of this cancer.

\section{Author contributions}

Conceptualization-SKS, PMS, PKV; Formal analysis and investigation-PMS, SKS, PP; Writing - original draft preparation-PMS, AS, PP, NC; Writing - review and editing-SKS; Resources-PMS, AS, PP, SKS; Supervision-SKS, PKV, AKS, BP.

\section{Ethics approval and consent to participate}

Not applicable.

\section{Acknowledgment}

Pingali M. Shivapriya and Anirudh Singh are thankful to MHRD, Govt. of India for fellowship. We are thankful to IIIT Allahabad for providing research facility. We are thankful to Arka Banerjee at department of Microbiology and Immunology, Weill Cornell Medicine and Ayush Amod, Dept. of Applied Sciences, IIITA for critical reading of the manuscript.

\section{Funding}

This research received no external funding.

\section{Conflict of interest}

The authors declare no conflict of interest.

\section{References}

[1] Kalemkerian GP, Akerley W, Bogner P, Borghaei H, Chow LQ, Downey RJ, et al. Small Cell Lung Cancer. Journal of the National Comprehensive Cancer Network. 2013; 11: 78-98.

[2] van Meerbeeck JP, Fennell DA, De Ruysscher DKM. Small-cell lung cancer. Lancet. 2011; 378: 1741-1755.

[3] Gaspar LE, McNamara EJ, Gay EG, Putnam JB, Crawford J, Herbst RS, et al. Small-cell lung cancer: prognostic factors and changing treatment over 15 years. Clinical Lung Cancer. 2012; 13: 115-122.

[4] Pleasance ED, Stephens PJ, O’Meara S, McBride DJ, Meynert $\mathrm{A}$, Jones $\mathrm{D}$, et al. A small-cell lung cancer genome with complex signatures of tobacco exposure. Nature. 2010; 463: 184-190.

[5] Sutherland KD, Proost N, Brouns I, Adriaensen D, Song J, Berns A. Cell of origin of small cell lung cancer: inactivation of Trp53 and Rb1 in distinct cell types of adult mouse lung. Cancer Cell. 2011; 19: 754-764.

[6] Byers LA, Rudin CM. Small cell lung cancer: where do we go from here? Cancer. 2015; 121: 664-672.

[7] Demedts IK, Vermaelen KY, van Meerbeeck JP. Treatment of extensive-stage small cell lung carcinoma: current status and future prospects. The European Respiratory Journal. 2010; 35: 202-215.

[8] Koinis F, Kotsakis A, Georgoulias V. Small cell lung cancer (SCLC): no treatment advances in recent years. Translational Lung Cancer Research. 2016; 5: 39-50.

[9] Semenova EA, Nagel R, Berns A. Origins, genetic landscape, and emerging therapies of small cell lung cancer. Genes \& Development. 2015; 29: 1447-1462.

[10] Kalemkerian GP. Small Cell Lung Cancer. Seminars in Respiratory and Critical Care Medicine. 2016; 37: 783-796.

[11] Yin X, Yan D, Qiu M, Huang L, Yan S. Prophylactic cranial irradiation in small cell lung cancer: a systematic review and metaanalysis. BMC Cancer. 2019; 19: 95.

[12] Wang S, Zimmermann S, Parikh K, Mansfield AS, Adjei AA. Current Diagnosis and Management of Small-Cell Lung Cancer. Mayo Clinic Proceedings. 2019; 94: 1599-1622.

[13] Park K, Martelotto LG, Peifer M, Sos ML, Karnezis AN, Mahjoub MR, et al. A crucial requirement for Hedgehog signaling in small cell lung cancer. Nature Medicine. 2011; 17: 15041508.

[14] Borah A, Raveendran S, Rochani A, Maekawa T, Kumar DS Targeting self-renewal pathways in cancer stem cells: clinical implications for cancer therapy. Oncogenesis. 2015; 4: e177.

[15] Pietanza MC, Byers LA, Minna JD, Rudin CM. Small Cell Lung Cancer: will Recent Progress Lead to Improved Outcomes? Clinical Cancer Research. 2015; 21: 2244-2255.

[16] Jackman DM, Johnson BE. Small-cell lung cancer. Lancet. 2005; 366: 1385-1396.

[17] Gazdar AF, Savage TK, Johnson JE, Berns A, Sage J, Linnoila RI, et al. The comparative pathology of genetically engineered mouse models for neuroendocrine carcinomas of the lung. Journal of Thoracic Oncology. 2015; 10: 553-564.

[18] Dempke WCM, Fenchel K, Uciechowski P, Chevassut T. Targeting Developmental Pathways: the Achilles Heel of Cancer? Oncology. 2017; 93: 213-223.

[19] Nwabo Kamdje AH, Takam Kamga P, Tagne Simo R, Vecchio L, Seke Etet PF, Muller JM, et al. Developmental pathways associated with cancer metastasis: Notch, Wnt, and Hedgehog. Cancer Biology \& Medicine. 2017; 14: 109-120.

[20] Gardner EE, Lok BH, Schneeberger VE, Desmeules P, Miles LA, Arnold PK, et al. Chemosensitive Relapse in Small Cell Lung Cancer Proceeds through an EZH2-SLFN11 Axis. Cancer Cell. 2017; 31: 286-299.

[21] Fernandes-Silva H, Correia-Pinto J, Moura RS. Canonical Sonic Hedgehog Signaling in Early Lung Development. Journal of Developmental Biology. 2017; 5: 3.

[22] Watkins DN, Berman DM, Burkholder SG, Wang B, Beachy PA, Baylin SB. Hedgehog signalling within airway epithelial pro- 
genitors and in small-cell lung cancer. Nature. 2003; 422: 313317.

[23] Ingham PW, McMahon AP. Hedgehog signaling in animal development: paradigms and principles. Genes \& Development. 2001; 15: 3059-3087.

[24] Gonnissen A, Isebaert S, Haustermans K. Targeting the Hedgehog signaling pathway in cancer: beyond Smoothened. Oncotarget. 2015; 6: 13899-13913.

[25] Mastrangelo E, Milani M. Role and inhibition of GLI1 protein in cancer. Lung Cancer. 2018; 9: 35-43.

[26] Taipale J, Beachy PA. The Hedgehog and Wnt signalling pathways in cancer. Nature. 2001; 411: 349-354.

[27] Watkins DN, Berman DM, Baylin SB. Hedgehog signaling: progenitor phenotype in small-cell lung cancer. Cell Cycle. 2003; 2: 196-198.

[28] Voortman J, Lee J, Killian JK, Suuriniemi M, Wang Y, Lucchi $\mathrm{M}$, et al. Array comparative genomic hybridization-based characterization of genetic alterations in pulmonary neuroendocrine tumors. Proceedings of the National Academy of Sciences of the United States of America. 2010; 107: 13040-13045.

[29] Chen JK, Taipale J, Cooper MK, Beachy PA. Inhibition of Hedgehog signaling by direct binding of cyclopamine to Smoothened. Genes \& Development. 2002; 16: 2743-2748.

[30] Von Hoff DD, LoRusso PM, Rudin CM, Reddy JC, Yauch RL, Tibes R, et al. Inhibition of the Hedgehog Pathway in Advanced Basal-Cell Carcinoma. New England Journal of Medicine. 2009; 361: 1164-1172.

[31] Belani CP, Dahlberg SE, Rudin CM, Fleisher M, Chen HX, Takebe $\mathrm{N}$, et al. Vismodegib or cixutumumab in combination with standard chemotherapy for patients with extensive-stage small cell lung cancer: a trial of the ECOG-ACRIN Cancer Research Group (E1508). Cancer. 2016; 122: 2371-2378.

[32] Kaur G, Reinhart RA, Monks A, Evans D, Morris J, Polley E, et al. Bromodomain and hedgehog pathway targets in small cell lung cancer. Cancer Letters. 2016; 371: 225-239.

[33] Kuramoto T, Goto H, Mitsuhashi A, Tabata S, Ogawa H, Uehara $\mathrm{H}$, et al. Dll4-Fc, an inhibitor of Dll4-notch signaling, suppresses liver metastasis of small cell lung cancer cells through the downregulation of the NF-kB activity. Molecular Cancer Therapeutics. 2012; 11: 2578-2587.

[34] Pietanza MC, Spira AI, Jotte RM, Gadgeel SM, Mita AC, Hart LL, et al. Final results of phase Ib of tarextumab (TRXT, OMP59R5, anti-Notch2/3) in combination with etoposide and platinum (EP) in patients (pts) with untreated extensive-stage smallcell lung cancer (ED-SCLC). Journal of Clinical Oncology. 2015; 33: 7508-7508.

[35] Isobe Y, Sato K, Nishinaga Y, Takahashi K, Taki S, Yasui H, et al. Near infrared photoimmunotherapy targeting DLL3 for small cell lung cancer. EBioMedicine. 2020; 52: 102632.

[36] Pardo OE, Latigo J, Jeffery RE, Nye E, Poulsom R, SpencerDene $\mathrm{B}$, et al. The fibroblast growth factor receptor inhibitor PD173074 blocks small cell lung cancer growth in vitro and in vivo. Cancer Research. 2009; 69: 8645-8651.

[37] Bahleda R, Italiano A, Hierro C, Mita A, Cervantes A, Chan N, et al. Multicenter Phase i Study of Erdafitinib (JNJ-42756493), Oral Pan-Fibroblast Growth Factor Receptor Inhibitor, in $\mathrm{Pa}$ tients with Advanced or Refractory Solid Tumors. Clinical Cancer Research. 2019; 25: 4888-4897.

[38] Pan F, Shen F, Yang L, Zhang L, Guo W, Tian J. Inhibitory effects of XAV939 on the proliferation of small-cell lung cancer H446 cells and $\mathrm{Wnt} / \beta$-catenin signaling pathway in vitro. Oncology Letters. 2018; 16: 1953-1958.

[39] Aditya S, Rattan A. Vismodegib: a smoothened inhibitor for the treatment of advanced basal cell carcinoma. Indian Dermatology Online Journal. 2013; 4: 365-368.

[40] Pietanza MC, Krug LM, Varghese AM, Fleisher M, Teitcher JB, Holodny A, et al. Phase I trial of the hedgehog (Hh) inhibitor, LDE225, in combination with etoposide and cisplatin (EP) for initial treatment of extensive stage small cell lung cancer (ESSCLC). Journal of Clinical Oncology. 2014; 32: 7602-7602.
[41] Pan S, Wu X, Jiang J, Gao W, Wan Y, Cheng D, et al. Discovery of NVP-LDE225, a Potent and Selective Smoothened Antagonist. ACS Medicinal Chemistry Letters. 2010; 1: 130-134.

[42] Hyman JM, Firestone AJ, Heine VM, Zhao Y, Ocasio CA, Han $\mathrm{K}$, et al. Small-molecule inhibitors reveal multiple strategies for Hedgehog pathway blockade. Proceedings of the National Academy of Sciences of the United States of America. 2009; 106: 14132-14137.

[43] Ferruzzi P, Mennillo F, De Rosa A, Giordano C, Rossi M, Benedetti $G$, et al. In vitro and in vivo characterization of a novel Hedgehog signaling antagonist in human glioblastoma cell lines. International Journal of Cancer. 2012; 131: E33-E44.

[44] Saunders LR, Bankovich AJ, Anderson WC, Aujay MA, Bheddah S, Black K, et al. A DLL3-targeted antibody-drug conjugate eradicates high-grade pulmonary neuroendocrine tumorinitiating cells in vivo. Science Translational Medicine. 2015; 7: 302ra136.

[45] Muller HJ. Types of visible variations induced by X-rays in Drosophila. Journal of Genetics. 1930; 22: 299-334.

[46] D’Souza B, Meloty-Kapella L, Weinmaster G. Canonical and non-canonical Notch ligands. Current Topics in Developmental Biology. 2010; 92: 73-129.

[47] Lin S, Negulescu A, Bulusu S, Gibert B, Delcros J, Ducarouge $\mathrm{B}$, et al. Non-canonical NOTCH3 signalling limits tumour angiogenesis. Nature Communications. 2017; 8: 16074.

[48] Ikezawa Y, Sakakibara-Konishi J, Mizugaki H, Oizumi S, Nishimura M. Inhibition of Notch and HIF enhances the antitumor effect of radiation in Notch expressing lung cancer. International Journal of Clinical Oncology. 2017; 22: 59-69.

[49] Ishibashi M, Ang SL, Shiota K, Nakanishi S, Kageyama R, Guillemot F. Targeted disruption of mammalian hairy and Enhancer of split homolog-1 (HES-1) leads to up-regulation of neural helix-loop-helix factors, premature neurogenesis, and severe neural tube defects. Genes \& Development. 1995; 9: 31363148.

[50] Borges M, Linnoila RI, van de Velde HJ, Chen H, Nelkin BD, Mabry M, et al. An achaete-scute homologue essential for neuroendocrine differentiation in the lung. Nature. 1997; 386: 852855.

[51] Rajasinghe LD, Gupta SV. Tocotrienol-rich mixture inhibits cell proliferation and induces apoptosis via down-regulation of the Notch-1/NF-\&kappa;B pathways in NSCLC cells. Nutrition and Dietary Supplements. 2017; 9: 103-114.

[52] Liu Z, Wu T, Li Q, Wang M, Jing L, Ruan Z, et al. Notch Signaling Components: Diverging Prognostic Indicators in Lung Adenocarcinoma. Medicine. 2016; 95: e3715.

[53] D’Souza B, Meloty-Kapella L, Weinmaster G. Canonical and non-canonical Notch ligands. Current topics in developmental biology. 2010; 92: 73-129.

[54] Chapman G, Sparrow DB, Kremmer E, Dunwoodie SL. Notch inhibition by the ligand DELTA-LIKE 3 defines the mechanism of abnormal vertebral segmentation in spondylocostal dysostosis. Human Molecular Genetics. 2011; 20: 905-916.

[55] Henke RM, Meredith DM, Borromeo MD, Savage TK, Johnson JE. Ascl1 and Neurog2 form novel complexes and regulate Delta-like3 (Dll3) expression in the neural tube. Developmental Biology. 2009; 328: 529-540.

[56] Leonetti A, Facchinetti F, Minari R, Cortellini A, Rolfo CD, Giovannetti E, et al. Notch pathway in small-cell lung cancer: from preclinical evidence to therapeutic challenges. Cellular Oncology. 2019; 42: 261-273.

[57] George J, Lim JS, Jang SJ, Cun Y, Ozretić L, Kong G, et al. Comprehensive genomic profiles of small cell lung cancer. Nature. 2015; 524: 47-53.

[58] Sriuranpong V, Borges MW, Ravi RK, Arnold DR, Nelkin BD, Baylin SB, et al. Notch signaling induces cell cycle arrest in small cell lung cancer cells. Cancer Research. 2001; 61: 32003205.

[59] Hassan WA, Yoshida R, Kudoh S, Kameyama H, Hasegawa K, Niimori-Kita K, et al. Notch1 controls cell chemoresistance in 
small cell lung carcinoma cells. Thoracic Cancer. 2016; 7: 123128.

[60] Wael H, Yoshida R, Kudoh S, Hasegawa K, Niimori-Kita K, Ito T. Notch1 signaling controls cell proliferation, apoptosis and differentiation in lung carcinoma. Lung Cancer. 2014; 85: 131140.

[61] Hassan WA, Yoshida R, Kudoh S, Motooka Y, Ito T. Evaluation of role of Notch3 signaling pathway in human lung cancer cells. Journal of Cancer Research and Clinical Oncology. 2016; 142: 981-993.

[62] Meder L, Büttner R, Odenthal M. Notch signaling triggers the tumor heterogeneity of small cell lung cancer. Journal of Thoracic Disease. 2017; 9: 4884-4888.

[63] Lim JS, Ibaseta A, Fischer MM, Cancilla B, O’Young G, Cristea $\mathrm{S}$, et al. Intratumoural heterogeneity generated by Notch signalling promotes small-cell lung cancer. Nature. 2017; 545: 360-364.

[64] Calbo J, Meuwissen R, Montfort EV, Tellingen OV, Berns A. Genotype-Phenotype Relationships in a Mouse Model for Human Small-Cell Lung Cancer. Cold Spring Harbor Symposia on Quantitative Biology. 2005; 70: 225-232.

[65] Rudin CM, Pietanza MC, Bauer TM, Ready N, Morgensztern D, Glisson BS, et al. Rovalpituzumab tesirine, a DLL3-targeted antibody-drug conjugate, in recurrent small-cell lung cancer: a first-in-human, first-in-class, open-label, phase 1 study. The Lancet Oncology. 2017; 18: 42-51.

[66] Morgensztern D, Besse B, Greillier L, Santana-Davila R, Ready N, Hann CL, et al. Efficacy and Safety of Rovalpituzumab Tesirine in third-Line and beyond Patients with DLL3-Expressing, RelapsedRefractory Small-Cell Lung Cancer: Results from the Phase II TRINITY Study. Clinical Cancer Research. 2019; 25: 6958-6966.

[67] Turner N, Grose R. Fibroblast growth factor signalling: from development to cancer. Nature Reviews. Cancer. 2010; 10: 116129.

[68] Wesche J, Haglund K, Haugsten EM. Fibroblast growth factors and their receptors in cancer. The Biochemical Journal. 2011; 437: 199-213.

[69] Kouhara H, Hadari YR, Spivak-Kroizman T, Schilling J, BarSagi D, Lax I, et al. A lipid-anchored Grb2-binding protein that links FGF-receptor activation to the Ras/MAPK signaling pathway. Cell. 1997; 89: 693-702.

[70] Klint P, Claesson-Welsh L. Signal transduction by fibroblast growth factor receptors. Frontiers in Bioscience. 1999; 4: D165D177.

[71] Eswarakumar VP, Lax I, Schlessinger J. Cellular signaling by fibroblast growth factor receptors. Cytokine \& Growth Factor Reviews. 2005; 16: 139-149.

[72] Ornitz DM, Itoh N. The Fibroblast Growth Factor signaling pathway. Wiley Interdisciplinary Reviews-Developmental Biology. 2015; 4: 215-266.

[73] Peifer M, Fernández-Cuesta L, Sos ML, George J, Seidel D, Kasper LH, et al. Integrative genome analyses identify key somatic driver mutations of small-cell lung cancer. Nature Genetics. 2012; 44: 1104-1110.

[74] Thomas A, Lee J, Abdullaev Z, Park K, Pineda M, Saidkhodjaeva L, et al. Characterization of Fibroblast Growth Factor Receptor 1 in Small-Cell Lung Cancer. Journal of Thoracic Oncology. 2014; 9: 567-571.

[75] Zhang L, Yu H, Badzio A, Boyle TA, Schildhaus H, Lu X, et $a l$. Fibroblast Growth Factor Receptor 1 and Related Ligands in Small-Cell Lung Cancer. Journal of Thoracic Oncology. 2015; 10: 1083-1090.

[76] Dieci MV, Arnedos M, Andre F, Soria JC. Fibroblast growth factor receptor inhibitors as a cancer treatment: from a biologic rationale to medical perspectives. Cancer Discovery. 2013; 3: 264-279.

[77] Liu F, Li N, Zhang Y, Xie W, Yang S, Lu T, et al. Recent advance in the development of novel, selective and potent FGFR in- hibitors. European Journal of Medicinal Chemistry. 2020; 186 111884.

[78] Desai A, Adjei AA. FGFR Signaling as a Target for Lung Cancer Therapy. Journal of Thoracic Oncology. 2016; 11: 9-20.

[79] Wagner AH, Devarakonda S, Skidmore ZL, Krysiak K, Ramu A, Trani L, et al. Recurrent WNT pathway alterations are frequent in relapsed small cell lung cancer. Nature Communications. 2018; 9: 3787.

[80] Esteller L, Hernández S, Lopez-Rios F, Remon J. Could WNT inhibitors really knock on the treatment door of small cell lung cancer? Journal of Thoracic Disease. 2019; 11: S381-S384.

[81] Chen S, Xu Y, Chen Y, Li X, Mou W, Wang L, et al. SOX2 gene regulates the transcriptional network of oncogenes and affects tumorigenesis of human lung cancer cells. PLoS ONE. 2012; 7: e36326.

[82] Saito A, Horie M, Nagase T. TGF- $\beta$ Signaling in Lung Health and Disease. International Journal of Molecular Sciences. 2018; 19: 2460.

[83] Wu L, Wang P. Long non-coding RNA-neighboring enhancer of FOXA2 inhibits the migration and invasion of small cell lung carcinoma cells by downregulating transforming growth factor$\beta 1$. Oncology Letters. 2019; 17: 4969-4975.

[84] Cao J, Song Y, Bi N, Shen J, Liu W, Fan J, et al. DNA methylation-mediated repression of miR-886-3p predicts poor outcome of human small cell lung cancer. Cancer Research. 2013; 73: 3326-3335.

[85] Guo L, Liu Y, Bai Y, Sun Y, Xiao F, Guo Y. Gene expression profiling of drug-resistant small cell lung cancer cells by combining microRNA and cDNA expression analysis. European Journal of Cancer. 2010; 46: 1692-1702.

[86] Tang R, Lei Y, Hu B, Yang J, Fang S, Wang Q, et al. WW domain binding protein 5 induces multidrug resistance of small cell lung cancer under the regulation of miR-335 through the Hippo pathway. British Journal of Cancer. 2016; 115: 243-251.

[87] Yang K, Zhao Y, Du Y, Tang R. Evaluation of Hippo Pathway and CD133 in Radiation Resistance in Small-Cell Lung Cancer. Journal of Oncology. 2021; 2021: 1-8.

[88] Han Y, Liu D, Li L. PD-1/PD-L1 pathway: current researches in cancer. American Journal of Cancer Research. 2020; 10: 727742.

[89] Castagnoli L, Cancila V, Cordoba-Romero SL, Faraci S, Talarico G, Belmonte B, et al. WNT signaling modulates PD-L1 expression in the stem cell compartment of triple-negative breast cancer. Oncogene. 2019; 38: 4047-4060.

[90] Chakrabarti J, Holokai L, Syu L, Steele NG, Chang J, Wang J, et al. Hedgehog signaling induces PD-L1 expression and tumor cell proliferation in gastric cancer. Oncotarget. 2018; 9: 37439 37457.

Keywords: Small cell lung cancer; Therapeutics; Signalling pathways; Notch pathway; FGF pathway; Hedgehog pathway

\section{Send correspondence to:}

Biswaranjan Paital, Redox Regulation Laboratory, Department of Zoology, Odisha University of Agriculture and Technology, College of Basic Science and Humanities, 751003 Bhubaneswar, Odisha, India, E-mail: Biswaranjanpaital@gmail.com

Pritish Kumar Varadwaj, Department of Applied Sciences, Indian Institute of Information Technology Allahabad, 211012 Allahabad, India, E-mail: pritish@iiita.ac.in

Sintu Kumar Samanta, Department of Applied Sciences, Indian Institute of Information Technology Allahabad, 211012 Allahabad, India, E-mail: samantasintu@iiita.ac.in 